\title{
Cerebral Blood Flow in Immediate and Sustained Anxiety
}

\author{
Gregor Hasler, ${ }^{1}$ Stephen Fromm, ${ }^{2}$ Ruben P. Alvarez, ${ }^{2}$ David A. Luckenbaugh, ${ }^{2}$ Wayne C. Drevets, ${ }^{2}$ and Christian Grillon ${ }^{2}$ \\ ${ }^{1}$ Psychiatric University Hospital, 8091 Zurich, Switzerland, and ${ }^{2}$ Mood and Anxiety Disorders Program, Intramural Research Program, National Institute of \\ Mental Health, National Institutes of Health, Bethesda, Maryland 20892
}

The goal of this study was to compare cerebral blood flow (CBF) changes associated with phasic cued fear versus those associated with sustained contextual anxiety. Positron emission tomography images of $\mathrm{CBF}$ were acquired using [0-15] $\mathrm{H}_{2} \mathrm{O}$ in 17 healthy human subjects as they anticipated unpleasant electric shocks that were administered predictably (signaled by a visual cue) or unpredictably (threatened by the context). Presentation of the cue in either threat condition was associated with increased CBF in the left amygdala. A cue that specifically predicted the shock was associated with CBF increases in the ventral prefrontal cortex (PFC), hypothalamus, anterior cingulate cortex, left insula, and bilateral putamen. The sustained threat context increased CBF in the right hippocampus, mid-cingulate gyrus, subgenual PFC, midbrain periaqueductal gray, thalamus, bilateral ventral striatum, and parieto-occipital cortex. This study showed distinct neuronal networks involved in cued fear and contextual anxiety underlying the importance of this distinction for studies on the pathophysiology of anxiety disorders.

Key words: fear; anxiety; amygdala; hippocampus; context; cerebral blood flow

\section{Introduction}

Understanding the nature of fear and anxiety provides a framework for interpreting neural responses to threat. This is of clinical relevance because fear and anxiety may represent different aspects of anxiety disorders. Specific phobia involves abnormal phasic responses to specific threat cues, whereas in panic disorders, generalized anxiety disorder, and depression, anxiety is more sustained and less linked to a specific cue (Grillon and Baas, 2003). Sustained responses to contextual cues and fear of specific threat cues may coexist in posttraumatic stress disorder (Pole et al., 2003).

Fear is linked to action tendency; it is a basic defensive response that prepares the organism to deal with an imminent threat. It is characterized by an impulse to escape or fight and typically results in a surge of sympathetic arousal. Fear is highly organized and relies on the operation of a phylogenetically older amygdalocentric limbic system subserving detection and immediate reactions to danger. Fear involves the recruitment and coordination of cognitive, motor, autonomic, and endocrine systems (Lang et al., 2000; Davis, 2004; Phelps and LeDoux, 2005). Empirical evidence has confirmed that the basolateral amygdala orchestrates cued fear processing. It receives sensory input from the cortex and the thalamus and organizes fear responses via anatomical projections to a network underlying fear-related memory, reflex, and endocrine responses including the central nucleus of the amygdala, hypothalamus, brain stem nuclei, striatum, and medial and orbital prefrontal cortices (Davis, 2004;

Received Dec. 13, 2006; revised April 30, 2007; accepted April 30, 2007.

This work was supported by the Intramural Research Program of the National Institute of Mental Health.

Correspondence should be addressed to Dr. Gregor Hasler, National Institutes of Health, National Institute of Mental Health, Mood and Anxiety Disorders Program, 15K North Drive, Room 200, MSC 2670, Bethesda, MD 208922670. E-mail: g.hasler@bluewin.ch.

DOI:10.1523/JNEUROSCI.5369-06.2007

Copyright $\odot 2007$ Society for Neuroscience $\quad$ 0270-6474/07/276313-07\$15.00/0
Phelps and LeDoux, 2005). These structures were hypothesized to be activated by cues predicting shocks in the present study.

The neurobiology of anxiety and related conditions associated with sustained aversive states is less well characterized. In contrast to fear, anxiety is less coordinated and organized. It is future oriented and entails more complex cognitive processing and symbolic representations of potential dangers. When a threat is present but not imminent, vigilance is heightened, but attention is not focused on a specific aspect of the environment, and sensory receptivity is facilitated. Although the role of amygdala in anxiety may be less important than in cued fear responses (Davis, 2004), anxiety may involve brain regions associated with (1) the detection of contextual threat such as the hippocampus (Kim and Fanselow, 1992; Phillips and LeDoux, 1992, 1995), which plays a crucial role in behavioral/anxious inhibition (Gray, 1982), (2) sustained defensive responses via structures such as the bed nucleus of the stria terminalis (Walker et al., 2003), and (3) the monitoring of the environment including the parietal cortex (Carlsson et al., 2006). Neural structures associated with contextual information, sustained defensive responses, and vigilance were expected to be activated by unpredictable shocks.

Most studies on the neurobiology of cued fear and sustained contextual anxiety have been conducted in rodents, which may differ considerably from humans in the neurobiology of fear circuits (Davis, 2004). The goal of this study was to identify brain regions in humans that are commonly and specifically involved in the processing of immediate fear and sustained anxiety using a paradigm that has been validated in clinical studies.

\section{Materials and Methods}

Subjects. Seventeen right-handed, medically and psychiatrically healthy volunteers [five females; age, $33 \pm 9.3$ (range, 20-47); Beck Anxiety Inventory total score, $0.8 \pm 1.0$ (range, $0-3$ ); Beck Depression Inventory total score, $0.4 \pm 0.8$ (range, $0-3$ )] participated in this study. Subjects were recruited through advertisements and evaluated during specific 
screening visits at the National Institutes of Health Clinical Center. The clinical evaluation consisted of physical examination, electrocardiography, and laboratory tests, including liver and kidney function tests, hematology profile, thyroid function tests, urinanalysis, and toxicology (drug screen). Pregnant or nursing women were excluded. Subjects denied exposure to psychotropic medications (including nicotine or any tobacco products) or other agents likely to influence cerebral blood flow $(\mathrm{CBF})$ or metabolism within the 1 month before scanning. Mental health status was evaluated by an unstructured clinical interview by a psychiatrist and by a structured interview using the Structured Clinical Interview for the Diagnostic and Statistical Manual of Mental Disorders, Non-patient Edition (First et al., 1996). Subjects provided written informed consent after receiving a full explanation of the study purpose, procedures, and risks, as approved by the National Institute of Mental Health Institutional Review Board.

Experimental design. The threat of unpleasant shocks administered predictably and unpredictably was implemented with the hypothesis that unpredictable, rather than predictable, aversive events evoke sustained anxious states (for review, see Grillon, 2002). In this paradigm, the aversive stimuli, described to the subjects as "unpleasant events," consisted of six electric shocks of $200 \mathrm{~ms}$ duration and ranged in intensity from 3.4 to $5.0 \mathrm{~mA}$. Shock intensity was determined individually using a shock workup procedure involving the administration of one to three sample shocks. Sample shocks began at a low-intensity level and were increased until a level was reached that subjects found unpleasant but not painful. Previous studies have shown that anticipating unpleasant shock is anxiogenic (Grillon et al., 2004). The shocks were delivered through two disk electrodes placed on the right forearm.

Figure 1 displays a schematic of the trial structure. Each trial followed an instructed threat procedure in which one of three conditions [neutral $(\mathrm{N})$, predictable $(\mathrm{P})$, or unpredictable $(\mathrm{U})$ ] was presented for $1.5 \mathrm{~min}$, not including the duration of the radiotracer transit time (see below, Positron emission tomography image acquisition and processing). Each condition was preceded and followed by $30 \mathrm{~s}$ of filler that was not included in analyses. The filler consisted of additional presentations of the $\mathrm{N}$ condition. In the $\mathrm{N}$ condition, no unpleasant events were delivered. In the $\mathrm{P}$ condition, unpleasant events were administered predictably, that is, only in the presence of a threat cue. In the $\mathrm{U}$ condition, unpleasant events were delivered at any time. During each trial, two 10-s-duration cues were presented. The cues consisted of different colored geometric shapes. In the $\mathrm{P}$ condition, the cues signaled the possibility of receiving an aversive stimulus, but they had no signal value in the $\mathrm{N}$ and $\mathrm{U}$ conditions. Throughout each trial, a computer monitor apprised participants of the current condition by displaying the following information: "No Unpleasant Event" for N, "Unpleasant Event Only During Red Square" for $\mathrm{P}$, or "Unpleasant Event at Any Time" for U. This information, in the absence of a cue, constituted the context for each condition. During each trial involving a $\mathrm{P}$ or $\mathrm{U}$ condition, one unpleasant event (shock) was administered. The shocks were delivered simultaneously with the offset of a cue in the $\mathrm{P}$ condition and in the absence of the cue in the $\mathrm{U}$ condition. Across subjects, shocks occurred an equal number of times during the early and late phases of a trial (e.g., shocks were administered to both the first and second cue of the P condition across subjects). Before the start of the experiment, participants were given an explanation of the study including explicit instructions regarding the conditions under which they would and would not receive an unpleasant shock.

During the experiment, stimuli were presented on a video monitor suspended above the scanner gantry. Two CBF images were acquired in each condition $(\mathrm{N}, \mathrm{P}, \mathrm{U})$ : once when the cue was displayed [neutral cue $(\mathrm{NQ})$, predictable cue (PQ), unpredictable cue (UQ)] and again when the cue was absent [neutral context (NX), predictable context (PX), unpredictable context (UX)] (i.e., during context only). Thus, there was one CBF scan per trial for a total of six CBF scans. Each of the six trials lasted $\sim 2.5$ min providing subjects several minutes of rest before the next run. The presentation of runs was counterbalanced so that within subjects, the $\mathrm{P}$ and $\mathrm{U}$ trials were equally likely to occur before or after an $\mathrm{N}$ trial and that across subjects, cue and context scans were equally likely to occur during the first and second halves of the experiment. The study was designed so that a CBF scan during a context began at least $20 \mathrm{~s}$ after the presentation of a cue. All scans began at least $25 \mathrm{~s}$ after the administration of a shock or occurred at least $40 \mathrm{~s}$ before any shock. At the end of each run, participants were asked to verbally rate their level of subjective anxiety elicited by the cue and context in each condition using Visual Analog Scales. A full factorial repeated-measures ANOVA was run with two stimulus and three condition levels. Greenhouse-Geisser $p$ values are reported because of concerns with sphericity based on Mauchly's test of sphericity. Least significant difference statistics were calculated post hoc to determine the specific location of omnibus effects. $p$ values are reported as significant at $p<0.05$, two-tailed.

Positron emission tomography image acquisition and processing. Subject preparation consisted of intravenous catheterization and immobilization of the head using a thermoplastic mask. Positron emission tomography (PET) scans were acquired as subjects rested with eyes watching a screen using a General Electric (Fairfield, CT) Advance scanner in threedimensional acquisition mode ( 35 contiguous slices with $4.25 \mathrm{~mm}$ plane separation; three-dimensional resolution, $6.4 \mathrm{~mm}$ full-width at halfmaximum). First, a transmission scan $(\sim 8 \mathrm{~min})$ was acquired using rotating rods of $68 \mathrm{Ge} / 68 \mathrm{Ga}$ with electronic windowing around the rods to minimize scatter to permit attenuation correction of the emission scans during the tracer uptake period (Huang et al., 1979). After the transmission scan, one resting CBF image was acquired after bolus injection of $10 \mathrm{mCi}$ of $[\mathrm{O}-15] \mathrm{H}_{2} \mathrm{O}$ to estimate the vascular transit time required for the radiotracer bolus to reach the brain for each subject. Because images obtained after bolus administration reflect the regional CBF pattern during the first 15-20 s after the tracer reached the brain (Hurtig et al., 1994), we then timed the task initiation to correspond to the arrival of the $[\mathrm{O}-15] \mathrm{H}_{2} \mathrm{O}$ bolus injection to the brain so that the 15-20 s window of sensitivity to flow changes started during the cue or context under study and in the absence of actual shock administration. Six additional $\mathrm{CBF}$ scans were acquired at $8 \mathrm{~min}$ interscan intervals using bolus injection of $10 \mathrm{mCi}$ of [O-15] $\mathrm{H}_{2} \mathrm{O}$ in the experimental conditions (three cue: NQ, PQ, UQ; three context: NX, PX, UX). Corrections for subject motion between the CBF scans were performed with a mutual information registration of each time static image to a standard frame before attenuation correction. Based on the calculated motion, the transmission images were resliced and projected for final attenuation correction, reconstruction, and realignment. The final reconstructed images were corrected for attenuation, scatter, random coincidences, dead time, and decay. Using Statistical Parametric Mapping software (SPM2; Wellcome Department of Imaging Neuroscience, London, UK), these images were coregistered to the structural magnetic resonance (MR) images, spatially normalized to the standardized spatial array of the Montreal Neurological Institute (MNI), and normalized to exclude global variations in blood flow. Each image was then smoothed using an $8 \mathrm{~mm}$ Gaussian kernel. A voxelwise analysis of the PET data were performed using SPM2 in the high-level mathematics environment Matlab 6 (The MathWorks, Natick, MA). The MNI coordinates derived from SPM2 were nonlinearly transformed to the stereotaxic coordinates of Talairach and Tournoux (1988) using the Matlab command "mni2tal" (http://imaging.mrc-cbu. cam.ac.uk/downloads/MNI2tal/mni2tal.m).

The regional hemodynamic changes associated with phasic cued fear and sustained contextual anxiety were identified by comparing the normalized $\mathrm{CBF}$ images between the threat and $\mathrm{N}$ conditions. We were mainly interested in similarities and differences of CBF between the contrast "PQ condition versus NQ condition" (PQ-NQ), and the contrast "UX condition versus NX condition" (UX-NX), reflecting neural activity during phasic fear and sustained anxiety, respectively. To evaluate the specificity of findings from the contrast analyses, we used conjunction analysis as implemented in SPM2 using the methods described by Nichols et al. (2005). To estimate correlations between CBF and selfreported anxiety levels during scanning, we used anxiety scores as covariates to detect positive and negative correlations between anxiety levels and $\mathrm{CBF}$ in voxelwise analyses. Because we found important differences in neural correlates between cue and context conditions, we applied these correlational analyses for cue and context conditions separately. We used uncorrected $p<0.001$ as the significance level for contrasts and correlational analyses and uncorrected $p<0.005$ for conjunction analyses. We chose a minimum cluster size of 10 voxels. 


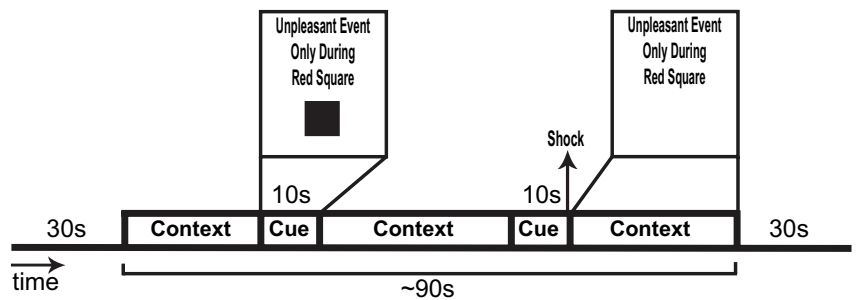

Figure 1. Example of $C B F$ image acquisition trial. Each trial followed an instructed threat procedure in which one of three conditions $(\mathrm{N}, \mathrm{P}$, or $\mathrm{U})$ was presented for $\sim 1.5 \mathrm{~min}$. Each condition was preceded and followed by $30 \mathrm{~s}$ of filler that was not included in analyses. In the $\mathrm{N}$ condition, no unpleasant events (shocks) were delivered. In the P condition (shown), unpleasant events were administered only in the presence of a threat cue. In the $U$ condition, unpleasant events were delivered at any time. During each trial, two 10-s-duration cues were presented. Cues consisted of different colored shapes (color omitted here). The cues signaled the possibility of receiving an aversive stimulus in the $P$ condition but had no signal value in the other conditions. Throughout each trial, a computer monitor apprised participants of the current condition by displaying one of the following messages: "No Unpleasant Event" (neutral), "Unpleasant Event Only During Red Square" (predictable), or "Unpleasant Event at Any Time" (unpredictable). This information, in the absence of a cue, constituted the context for each condition. One shock was administered during each trial involving a P or U condition. The shocks were delivered simultaneously with the offset of a cue in the $P$ condition and in the absence of the cue in the $U$ condition. Two CBF images were acquired in each condition: one when the cue was displayed along with the instructions and another when the cue was absent (i.e., during the context only). Thus, one (BF scan was acquired per trial.

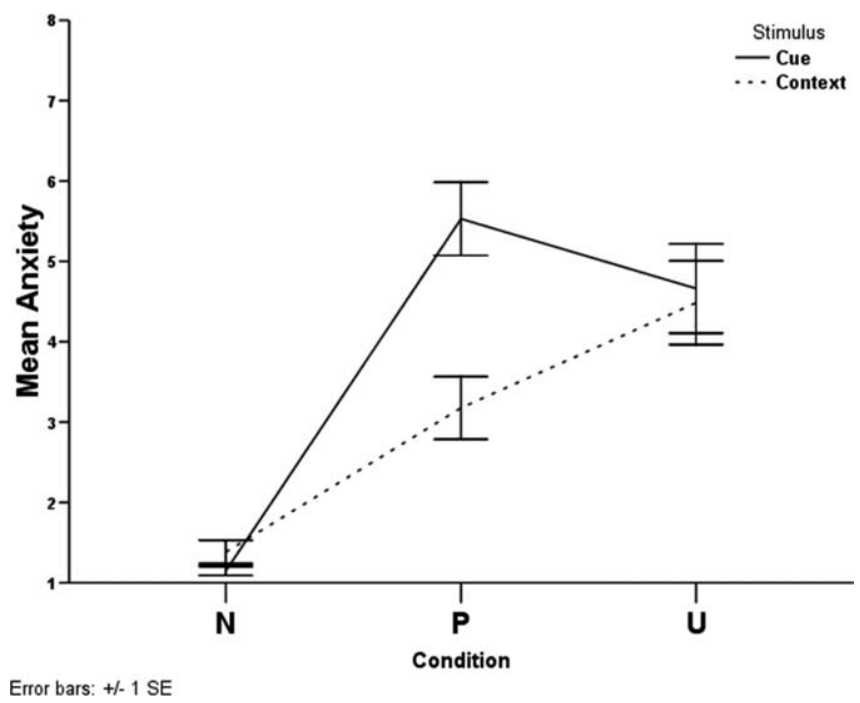

Figure 2. Self-reported anxiety levels classified by condition (N, P, U) and stimulus (cue, context).

\section{Results}

Figure 1 displays the design and the timeline associated with each trial; the experiment is described in more detail in Materials and Methods (see Experimental design). Figure 2 shows the average reported anxiety level classified in the no shock $(\mathrm{N})$, predictable shock $(\mathrm{P})$, and unpredictable shock $(\mathrm{U})$ conditions when the cue was present or absent (context). There were significant withinsubjects effects for condition $\left(F_{(1.7,27.6)}=36.61 ; p<0.0001\right)$ and stimulus $\left(F_{(1,16)}=40.87 ; p<0.0001\right)$ and a significant conditionby-stimulus interaction $\left(F_{(1.3,20.4)}=30.29 ; p<0.0001\right)$. Follow-up tests revealed that in the presence of the cue, anxiety levels in the $\mathrm{P}$ and the $\mathrm{U}$ conditions were higher than in the $\mathrm{N}$ condition ( $p<0.0001$ for both differences), and subjects were more anxious in the $\mathrm{P}$ than in the $\mathrm{U}$ condition $(p=0.024)$ (Fig. $1)$; in the absence of the cue (context), anxiety levels in the P and the $\mathrm{U}$ conditions were higher than in the $\mathrm{N}$ condition $(p<$ 0.0001 for both differences), and subjects were more anxious in the $\mathrm{U}$ than in the $\mathrm{P}$ condition $(p=0.026)$. Also, there was no difference in anxiety levels between cue and context for the $\mathrm{N}$ $(p=0.104)$ and $\mathrm{U}(p=0.269)$ conditions, but there was a significant difference between cue and context in the $\mathrm{P}$ condition $(p<0.001)$.

\section{Cue conditions}

Table 1 and Figure 3 display the results from the contrast analyses on cue conditions. Because the visual cues were not associated with any threat of shock in the NQ condition but predicted a potential shock in the PQ condition, the PQ-NQ contrast reflected increases in $\mathrm{CBF}$ associated with phasic fear. In the PQ-NQ contrast, CBF increased in the PQ condition relative to the NQ condition in the lateral and ventromedial prefrontal cortex (PFC), the vicinity of the hypothalamus, the right medial fusiform cortex, the right cerebellum, the left insula, the left amygdala, the medial parietal cortex/precuneus, the anterior cingulate cortex (ACC), and the bilateral putamen at uncorrected $p<0.001$.

In the UQ condition, the cue did not increase or decrease the risk of receiving a shock although the cue was presented during a period of unpredictable threat. The UQ-NQ contrast therefore reflected the $\mathrm{CBF}$ response to a cue not predictive of danger in a threatening condition. The UQ-NQ contrast showed increases in $\mathrm{CBF}$ in the right inferior parietal lobe, the right medial fusiform cortex, the left amygdala, the left interior temporal gyrus, the dorsomedial PFC, the right cuneus, the left anteroventral striatum, the left putamen, the left hippocampus, the mid-cingulate cortex, the right inferior frontal gyrus, and the bilateral cerebellum (all at uncorrected $p<0.001$ ). The conjunction analysis of the two contrasts (PQ-NQ and UQ-NQ) (Table 3 ) revealed areas where $\mathrm{CBF}$ increased in both contrasts in the ventral PFC, ACC, the left insula, and left and right striatum were specifically related to activation induced by a cue, whether the cue itself was predictive of a shock or was instead presented within a threat context.

The correlation analysis including the behavioral rating as covariate on the relationship between subjective anxiety levels and $\mathrm{CBF}$ changes in the cue conditions showed that hemodynamic activity was positively correlated to anxiety scores in the left putamen ( $x, y, z$ coordinates: $-28,4,2 ; t=5.31$; corrected cluster-level $p=0.02)$, the right and left cerebellum $(30,-80$, $-38 ; t=5.09 ; 42,-38,-25 ; t=4.73 ; 14,-66,-8 ; t=4.60)$, the right postcentral gyrus $(20,-38,61 ; t=4.55)$, the left ventrolateral PFC $(-30,46,-9 ; t=4.19)$, and the ACC $(-2,32,19 ; t=$ 4.08). Anxiety levels and CBF were inversely correlated in the right infralimbic cortex $(x, y, z$ coordinates: $14,3,-20 ; t=6.05)$, the right middle temporal gyrus $(61,-32,-14 ; t=5.89)$, the right dorsolateral PFC $(50,21,34 ; t=5.45)$, the left temporopolar cortex $(-40,16,-28 ; t=4.85)$, the left hippocampus $(-42$, $-39,6 ; t=4.19)$, and various regions of the occipital cortex.

\section{Context conditions}

Table 2 and Figure 3 show the results for the contrast analyses of the context conditions. Because the NX condition was safe and the UX condition was associated with unpredictably timed shocks, the UX-NX contrast reflected increases in CBF associated with sustained contextual anxiety. In the UX-NX contrast, CBF increased in the right cerebellum, the vicinity of the hypothalamus, the periaqueductal gray (PAG), the left precentral gyrus, the 
Table 1. PET blood flow changes in the cue conditions

\begin{tabular}{|c|c|c|c|c|c|}
\hline \multicolumn{3}{|l|}{$\mathrm{PQ}$ vs NQ } & \multicolumn{3}{|l|}{ UQ vs NQ } \\
\hline Brain regions & $x, y, z$ coordinates $^{a}$ & $t$ values & Brain regions & $x, y, z$ coordinates $^{a}$ & $t$ values \\
\hline L ventromedial PFC & $-8,48,-4$ & 3.92 & R inferior parietal lobe & $53,-41,26$ & 4.18 \\
\hline Vicinity of the hypothalamus & $-2,2,-8$ & 3.80 & R cerebellum & $6,-77,-26$ & 4.02 \\
\hline R medial fusiform cortex & $14,-67,-10$ & 3.50 & & $24,-58,-31$ & 3.54 \\
\hline Medial cerebellum & $6,-77,-26$ & 3.24 & & $42,-64,-27$ & 3.24 \\
\hline R lateral cerebellum & $32,-77,-20$ & 3.24 & & $34,-77,-20$ & 3.23 \\
\hline R ventrolateral PFC & $20,47,16$ & 3.47 & $\mathrm{R}$ medial fusiform cortex & $26,-49,-13$ & 3.72 \\
\hline L anterior insula & $-42,16,7$ & 3.43 & Lamygdala & $-28,-1,-10$ & 3.80 \\
\hline L amygdala & $-26,1,-14$ & 3.32 & L cerebellum & $-42,-58,-29$ & 3.44 \\
\hline Medial parietal cortex/precuneus & $4,-56,53$ & 3.28 & & $-2,-55,-19$ & 3.71 \\
\hline \multirow[t]{2}{*}{ Pregenual anterior cingulate cortex } & $2,32,19$ & 3.08 & & & \\
\hline & $-2,36,13$ & 2.94 & & $-22,-79,-23$ & 3.19 \\
\hline \multirow[t]{2}{*}{ R ventral putamen } & $20,4,5$ & 3.06 & L inferior temporal gyrus & $-50,-59,-14$ & 3.21 \\
\hline & & & Dorsomedial PFC & $4,11,62$ & 3.72 \\
\hline \multirow[t]{6}{*}{ L putamen } & $-24,8,14$ & 2.99 & R cuneus & $10,-96,10$ & 3.72 \\
\hline & & & L anteroventral striatum & $-20,10,-4$ & 3.50 \\
\hline & & & L ventral putamen & $-24,0,4$ & 3.32 \\
\hline & & & L hippocampus & $-36,-28,-7$ & 3.34 \\
\hline & & & Mid-cingulate gyrus & $-4,-17,40$ & 3.31 \\
\hline & & & R inferior frontal gyrus & $51,10,5$ & 3.23 \\
\hline
\end{tabular}

Regions were derived from contrast analyses comparing normalized CBF shown between two task conditions. All results were significant at uncorrected $p<0.001$. L, Left; R, right.

${ }^{a}$ Coordinates correspond to the stereotaxic array of Talairach and Tournoux (1988) and denote the distance in millimeters from the anterior commissure, with positive $x=$ right of midline, positive $y=$ anterior to the anterior commissure and positive $z=$ dorsal to a plane containing both the anterior and the posterior commissures.

right mid-cingulate cortex, the bilateral striatum, the right hippocampus/ventral pallidum, the subgenual PFC, and the right inferior parietal lobe at uncorrected $p<0.001$.

This and previous studies have shown that a context condition in which a shock is administered predictably (PX) also is anxiogenic, although to a lesser extent than an unpredictable context (Grillon et al., 2004). The PX-UX contrast showed increased CBF (uncorrected $p<0.001$ ) in the bilaterial striatum, the bilateral occipital cortex, the right hippocampus, the mid-cingulate cortex, the superior frontal gyrus on both sides, the medial thalamus, the right cerebellum, the left postcentral gyrus, the left ventrolateral PFC, and the left insula. The conjunction analysis of the two contrasts (UX-NX and PX-NX) (Table 3) revealed that $\mathrm{CBF}$ increased in the right cerebellum, subgenual $\mathrm{PFC}$, and the right inferior parietal lobe specifically during sustained contextual anxiety.

The correlation analysis including the behavioral rating as covariate on the relationship between subjective anxiety levels and $\mathrm{CBF}$ changes in the context conditions showed positive correlations in the left caudate nucleus $(x, y, z$ coordinates: $-14,12,12 ; t=6.66)$, the left cerebellum $(-22,-48,-18 ; t=6.57)$, the left midcingulate cortex $(-10,-14,39 ; t=5.43)$, the superior parietal lobe $(-8,-46,58$; $t=5.30)$, the right lateral orbital cortex $(46,23,-6 ; t=4.87)$, and the left anterior insula $(-32,8,-4 ; t=4.46)$. There was a negative correlation at cluster-level cor-
(A) IMMEDIATE ANXIETY (PREDICTABLE FEAR-CUE)

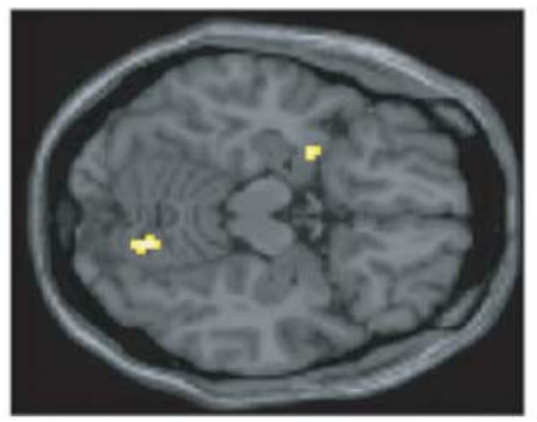

Horizontal view ( $z=-14)$ : left amygdala, right cerebellum

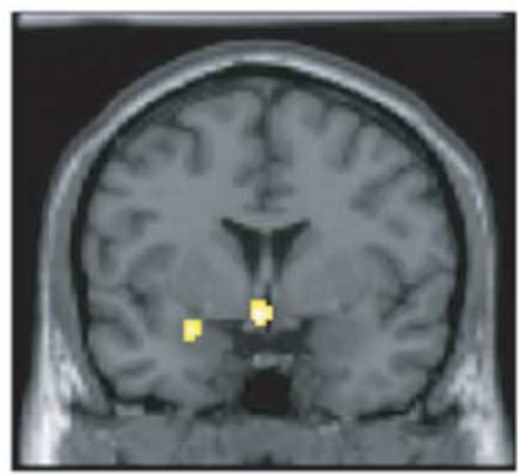

Coronal view $(z=1)$ : left amygdala, hypothalamus

\section{(B) SUSTAINED ANXIETY (THREAT CONTEXT)}

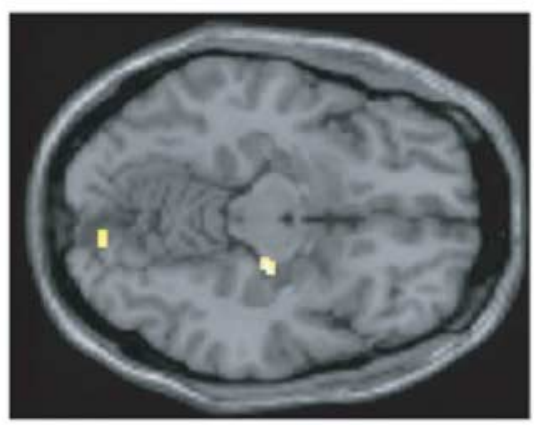

Horizontal view $(z=18)$ : right hippocampus, right cerebellum

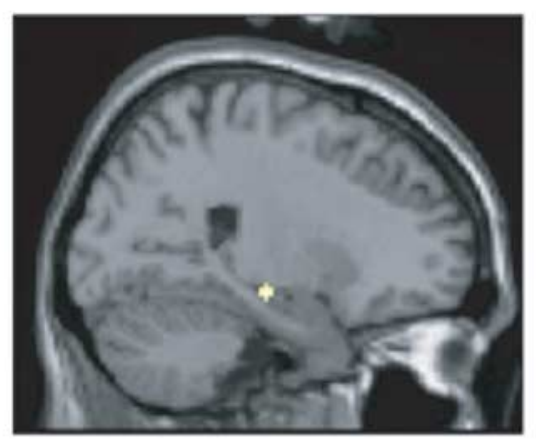

Sagittal view (right, $x+24)$ : right hippocampus
Figure 3. Merged PET-MRI sections illustrating CBF increases in mesiotemporal brain structures displayed on a canonical brain. $\boldsymbol{A}, \mathrm{CBF}$ activations during the cue predicting an electric shock at $p<0.005$. $\boldsymbol{B}, \mathrm{CBF}$ activations during the threat context at $p<$ 0.005 
Table 2. PET blood flow changes in the context conditions

\begin{tabular}{|c|c|c|c|c|c|}
\hline \multicolumn{3}{|l|}{ UXvs NX } & \multicolumn{3}{|l|}{ PXvs NX } \\
\hline Brain regions & $x, y, z$ coordinates & $t$ values & Brain regions & $x, y, z$ coordinates & $t$ values \\
\hline \multirow[t]{2}{*}{$\mathrm{R}$ cerebellum } & $12,-43,-13$ & 4.20 & L ventral putamen & $-22,-8,0$ & 4.14 \\
\hline & $14,-32,-22$ & 3.10 & R lingual gyrus & $12,-86,-11$ & 4.12 \\
\hline Vicinity of the hypothalamus & $-2,-12,-9$ & 4.17 & & $4,-82,-1$ & 3.27 \\
\hline Midbrain periaqueductal gray & $4,-26,-5$ & 3.44 & & $20,-87,12$ & 3.72 \\
\hline \multirow[t]{2}{*}{ L precentral gyrus } & $-8,-15,58$ & 4.11 & R hippocampus & $24,-18,-9$ & 4.10 \\
\hline & $-12,-12,41$ & 3.73 & Mid-cingulate gyrus & $4,-12,26$ & 4.04 \\
\hline R mid-cingulate gyrus & $8,-10,30$ & 4.10 & L superior frontal gyrus & $-12,-14,36$ & 3.96 \\
\hline R anteroventral striatum & $28,8,-2$ & 4.03 & R superior frontal gyrus & $12,19,38$ & 3.77 \\
\hline R hippocampus/ventral pallidum & $24,-16,-4$ & 3.14 & Medial thalamus & $0,-9,15$ & 3.77 \\
\hline Subgenual prefrontal cortex & $4,34,-10$ & 3.41 & R cerebellum & $18,-57,-12$ & 3.43 \\
\hline L caudate & $-14,14,9$ & 3.37 & & $44,-61,-20$ & 3.38 \\
\hline L anteroventral striatum & $-16,0,2$ & 3.30 & L postcentral gyrus & $-22,-34,68$ & 3.38 \\
\hline \multirow[t]{8}{*}{ R inferior parietal lobe } & $44,-39,42$ & 3.29 & L inferior occipital gyrus & $-30,-90,-11$ & 3.34 \\
\hline & & & Lingual gyrus & $-4,-91,0$ & 3.33 \\
\hline & & & & $-16,-86,-1$ & 3.25 \\
\hline & & & & $-12,-92,-7$ & 3.17 \\
\hline & & & R anteroventral striatum & $24,5,-7$ & 3.25 \\
\hline & & & R putamen & $24,-13,14$ & 3.18 \\
\hline & & & L ventrolateral PFC & $-26,45,12$ & 3.24 \\
\hline & & & L anterior insula & $-38,11,-7$ & 3.13 \\
\hline
\end{tabular}

Regions were derived from contrast analyses comparing normalized CBF shown between two task conditions. All results were significant at uncorrected $p<0.001$. Abbreviations and interpretation of stereotaxic coordinates are as in Table 1.

Table 3. Conjunction analyses of PET blood flow changes

\begin{tabular}{|c|c|c|c|c|c|}
\hline \multicolumn{3}{|c|}{ Conjunction of ( $\mathrm{PQ}$ vs NQ) and ( $\mathrm{UQ}$ vs NQ) } & \multicolumn{3}{|c|}{ Conjunction of (UX-NX) and (PX-NX) } \\
\hline Brain regions & $x, y, z$ coordinates & $t$ values & Brain regions & $x, y, z$ coordinates & $t$ values \\
\hline \multirow[t]{2}{*}{ Medial cerebellum } & $6,-77,-26$ & 3.36 & L precentral gyrus & $-12,-12,39$ & 3.59 \\
\hline & $-4,-57,-21$ & 2.66 & Mid-cingulate gyrus & $6,-8,30$ & 3.45 \\
\hline Postcentral gyrus & $-6,-43,72$ & 3.22 & Medial cerebellum & $4,-40,-15$ & 3.38 \\
\hline R cerebellum & $34,-79,-18$ & 3.13 & & $8,-84,-18$ & 2.87 \\
\hline L amygdala & $-26,-1,-12$ & 2.95 & R hippocampus/ventral pallidum & $24,-16,-6$ & 3.10 \\
\hline R inferior parietal lobe & $57,-45,24$ & 2.82 & L striatum (anterior caudate) & $-14,14,9$ & 3.05 \\
\hline Posterior cingulate cortex & $6,-45,32$ & 2.81 & R anterioventral striatum & $24,6,-5$ & 3.02 \\
\hline \multirow[t]{4}{*}{ Medial parietal cortex/precuneus } & $4,-54,54$ & 2.79 & R precentral gyrus & $61,4,7$ & 2.96 \\
\hline & & & L anterior insula & $-32,7,-10$ & 2.83 \\
\hline & & & & $-40,11,-6$ & 2.74 \\
\hline & & & Medial thalamus & $4,-9,15$ & 2.79 \\
\hline
\end{tabular}

Regions were derived from conjunction analyses on normalized CBF that included results from two contrast analyses. All results were significant at uncorrected $p<0.005$. Abbreviations and interpretation of stereotaxic coordinates are as in Table 1.

rected $p=0.01$ in the right inferior parietal lobe $(51,-46,54 ; t=$ 6.53) and additional negative correlations at uncorrected $p<$ 0.001 at various regions of the right and left inferior parietal lobes.

\section{Conjunction analysis of cue-mediated versus context-mediated fear}

The specificity of regions implicated in the cued versus contextual fear processing conditions was further assessed using a conjunction analysis of the predictable cue condition (PQ-NQ) and the unpredictable context condition (UX-NX). This analysis did not show any regions where increases in $\mathrm{CBF}$ were common to the two contrasts at $p_{\text {uncorrected }}<0.001$.

\section{Discussion}

Consistent with our hypotheses, presenting a cue in a threat condition was associated with increased CBF in the left amygdala; the specific association between the cue and a short immediate threat was associated with CBF increases in the ventral PFC, the hypothalamus, and the striatum. Sustained anxiety in the threat contexts generated increased $\mathrm{CBF}$ in the right hippocampus/ventral pallidum and brain areas implicated in vigilance and attention including the thalamus and the parieto-occipital cortex. The context of an unpredictable, unconditioned threat specifically was associated with CBF increases in the subgenual PFC and the PAG.

This study shows that the threat-related activation of the amygdala was related to the exposure to the visual cue in the threat conditions regardless of shock predictability and subjective anxiety response. This is consistent with the view that the amygdala may serve as a first-stage stimulus relevance in humans, reacting to ambiguous stimuli of potential motivational significance (Davis, 2004; Kim et al., 2004). Other areas activated by the visual cue regardless of shock predictability included various regions of the posterior cingulate cortex, the parieto-occipital cortex, and the cerebellum. The posterior cingulate cortex appears to serve as a sensory association cortex and may participate in processing the affective salience of sensory stimuli (Charney and Drevets, 2002). The parieto-occipital cortex has been implicated in visual awareness and visual short-term memory (Driver and Mattingley, 1998; Xu and Chun, 2006), possibly being involved in the screening of visual stimuli. The cerebellar activation may reflect activity of nonmotor, closed-loop circuits between the cerebellum and the PFC (Kelly and Strick, 2003).

Responses to the visual threat cue in the $\mathrm{P}$ condition specifi- 
cally were paralleled by activation in the ventral PFC, the hypothalamus, the right medial fusiform cortex, the ACC, and the left and right ventral putamen. Activation of the medial fusiform gyrus may reflect cue perception because this region is associated with the recognition of objects (Haxby et al., 1999) and previous research suggested that emotional stimuli activate inferotemporal visual areas more than non-emotional stimuli (Sabatinelli et al., 2005), possibly related to a direct path from the amygdala, which highlights the relevance of emotion in directing attention and perceptual processing. Activation of the hypothalamus may reflect the autonomic and neuroendocrine aspects of the stress response. The medial and ventrolateral PFC, the anteroventral striatum, the superior temporal sulcus, and certain regions of the insular cortex form an anatomical "viscero-motor" network that modulates visceral activity in response to affective stimuli, providing the major cortical output to visceromotor structures in the striatum, hypothalamus, and brainstem (Ongur and Price, 2000). Activation of these structures is consistent with the view that cued fear prepares the organism to react behaviorally (i.e., flight or fight) to an imminent danger. The striatal activation in this study is in line with previous work demonstrating direct activation of the ventral striatum in anticipation of aversive stimuli (Jensen et al., 2003). It has been proposed that the striatum and its major dopaminergic inputs may play a role by providing a signal that facilitates the reallocation of resources toward important, salient events (Zink et al., 2006). The negative correlation between cuerelated anxiety levels and $\mathrm{CBF}$ in the infralimbic cortex is consistent with studies in rats (Milad and Quirk, 2002) demonstrating that pairing conditioned threat cues with brief infralimbic stimulation reduced fear.

Sustained contextual anxiety in the $\mathrm{P}$ and $\mathrm{U}$ conditions, compared with the $\mathrm{N}$ condition, was associated with activation in the right hippocampus/ventral pallidum, the left and right striatum, the mid-cingulate cortex, the precentral gyrus, the thalamus, the left insula, the cerebellum, and the parieto-occipital cortex. There is abundant evidence for the role of the hippocampus in contextual anxiety. Electrolytic lesions of the hippocampus produce specific deficits in context conditioning in rats (Maren et al., 1997). Lesions of the hippocampus block freezing to the context although they do not impair stimulus-specific fear conditioning, suggesting that contextual anxiety requires both the amygdala and the hippocampus, whereas cued fear conditioning requires only the amygdala (Kim and Fanselow, 1992; Phillips and LeDoux, 1992; LeDoux, 2000). For acquisition of contextual anxiety, sensory stimuli enter the amygdala from the hippocampus via the entorhinal cortex (Phelps and LeDoux, 2005). Areas of the ventral hippocampus project to the basal amygdala, and damage to these areas interfered with contextual conditioning (Maren and Fanselow, 1995).

Pain studies show that the anticipation of aversive events activates regions within the medial PFC, the caudal anterior and mid-cingulate cortex, the thalamus, and cerebellum distinct from, but close to, locations mediating the experience of pain (Ploghaus et al., 1999; Koyama et al., 2005; Berns et al., 2006). In the current study, CBF in these brain regions was positively correlated with sustained anxiety levels. A recent functional MR imaging (MRI) study on pain processing (Carlsson et al., 2006) using predictable and unpredictable aversive stimuli found that predictable pain was associated with amygdala activation, whereas unpredictable pain was associated with increased activity in the fronto-parietal cortex and in the ventral striatum. In the present study, the activation of the parietal cortex in the unpredictable threat condition, the negative correlations between CBF in the inferior parietal cortex and anxiety levels, and the positive correlations between the superior parietal cortex and anxiety levels may reflect the involvement of attentional processes (e.g., vigilance) in the anticipation of unpredictable aversive events.

The subgenual PFC showed CBF increase in the unpredictable context condition. The subgenual PFC has reciprocal connections with areas implicated in the expression of behavioral, autonomic and endocrine responses to aversive stimuli, such as the lateral hypothalamus, amygdala, insula, accumbens, and PAG (for review, see Drevets et al., 1998). Human neuroimaging studies point to a critical role of the subgenual PFC in monitoring behaviorally salient stimuli (Berns et al., 2001). In rodents, lesions of infralimbic areas that correspond to the primate subgenual PFC lead to exaggerated responsiveness of the mesolimbic dopamine system to stress, including increased sympathetic arousal and corticosterone responses (Deutch et al., 1990; Sullivan and Gratton, 1999). Together, these results suggest that the subgenual PFC plays a sustained inhibitory role in stress-related visceral responses to unpredictable aversive events.

The lack of amygdala activation during sustained anxiety is not unexpected. In humans, amygdala response to threat cues habituate rapidly (Phelps et al., 2001). In addition, Davis (2004) suggested that although the amygdala initiates fear responses, during detection of a threat, the BNST is responsible for maintaining sustained responses. It is interesting that the bilateral destruction of the amygdala in primates led to blunting of acute fear responses, whereas trait-like sustained anxiety responses remained intact (Kalin et al., 2001). It is also possible that the temporal resolution of the imaging method used was too low to detect amygdala activation, which showed significant variability over time (Phillips et al., 2001).

These findings should be interpreted in the context of the strengths and limitations of this study. Human studies on the neurobiological substrates of anxiety have mostly relied on responses to specific stimuli (faces, conditioned cues), which may be relevant to certain anxiety types (e.g., phobias) but not others (e.g., generalized anxiety disorder). The strength of this study is that procedures involving responses to predictable and unpredictable aversive events model qualitatively distinct forms of aversive states, and have been well validated in psychophysiological, clinical, and psychopharmacological investigations (Grillon et al., 2004, 2006; Lissek et al., 2005). Another strength is that we used an experimental paradigm that can be replicated in animals, hence bridging the gap between preclinical and clinical research. A limitation of the study is that each trial was presented only a few times. However, we chose [O-15] $\mathrm{H}_{2} \mathrm{O}$ PET imaging because this method is sensitive enough to detect CBF changes in response to events that happen only a few times. In addition, few presentations of the threat periods reduce potential habituation to the mildly unpleasant aversive stimuli. Because the critical time epoch captured by each $\mathrm{CBF}$ scan only lasts $\sim 7 \mathrm{~s}$, the careful timing of the scans allowed for a temporal resolution that was adequate for the psychological paradigm examined in this study. However, other, highly temporal aspects of the difference between cue and context anxiety could not be evaluated with the imaging technique used. Because participants were fully informed about the cue-aversive event relationship, the study examined the expression for fear/anxiety rather than the acquisition of fear/anxiety.

Given the important role of fear and anxiety in major psychiatric disorders, the elucidation of neuronal networks mediating these aversive states in humans is highly informative. This study showed distinct neuronal networks involved in phasic fear and sustained anxiety. Of potential importance, brain regions that 
were associated with sustained anxiety such as the hippocampus and the subgenual PFC have been sites where structural and functional abnormalities have been demonstrated in previous neuroimaging studies of mood and anxiety disorders (Hasler et al., 2004). The neurobiological mechanisms mediating immediate and sustained anxiety may hold cues for better diagnostics and treatment of major pubic health conditions.

\section{References}

Berns GS, McClure SM, Pagnoni G, Montague PR (2001) Predictability modulates human brain response to reward. J Neurosci 21:2793-2798.

Berns GS, Chappelow J, Cekic M, Zink CF, Pagnoni G, Martin-Skurski ME (2006) Neurobiological substrates of dread. Science 312:754-758.

Carlsson K, Andersson J, Petrovic P, Petersson KM, Ohman A, Ingvar M (2006) Predictability modulates the affective and sensory-discriminative neural processing of pain. NeuroImage 32:1804-1814

Charney DS, Drevets WC (2002) Neurobiological basis of anxiety disorders. In: Neuropsychopharmacology: the fifth generation of progress (Davis KL, Charney DS, Coyle JT, Nemeroff CB, eds), pp 901-930. Philadelphia: Lippincott.

Davis M (2004) Functional neuroanatomy of anxiety and fear. In: Neurobiology of mental illness, Ed 2 (Charney DS, Nestler EJ, eds), pp 584-604. Oxford: Oxford UP.

Deutch AY, Clark WA, Roth RH (1990) Prefrontal cortical dopamine depletion enhances the responsiveness of mesolimbic dopamine neurons to stress. Brain Res 521:311-315.

Drevets WC, Ongur D, Price JL (1998) Neuroimaging abnormalities in the subgenual prefrontal cortex: implications for the pathophysiology of familial mood disorders. Mol Psychiatry 3:220- 226:190-221.

Driver J, Mattingley JB (1998) Parietal neglect and visual awareness. Nat Neurosci 1:17-22

First MB, Gibbon M, Spitzer RL, Williams JBW (1996) Structured clinical interview for DSM-IV axis I disorders: nonpatient edition (SCID-I/NP). New York: New York State Psychiatric Institute.

Gray JA (1982) The neuropsychology of anxiety: an enquiry in to the functions of the septo-hippocampal system. Oxford: Oxford UP.

Grillon C (2002) Startle reactivity and anxiety disorders: aversive conditioning, context, and neurobiology. Biol Psychiatry 52:958-975.

Grillon C, Baas J (2003) A review of the modulation of the startle reflex by affective states and its application in psychiatry. Clin Neurophysiol 114:1557-1579.

Grillon C, Baas JP, Lissek S, Smith K, Milstein J (2004) Anxious responses to predictable and unpredictable aversive events. Behav Neurosci 118:916-924.

Grillon C, Baas JM, Pine DS, Lissek S, Lawley M, Ellis V, Levine J (2006) The benzodiazepine alprazolam dissociates contextual fear from cued fear in humans as assessed by fear-potentiated startle. Biol Psychiatry 60:760-766.

Hasler G, Drevets WC, Manji HK, Charney DS (2004) Discovering endophenotypes for major depression. Neuropsychopharmacology 29:1765-1781.

Haxby JV, Ungerleider LG, Clark VP, Schouten JL, Hoffman EA, Martin A (1999) The effect of face inversion on activity in human neural systems for face and object perception. Neuron 22:189-199.

Huang SC, Hoffman EJ, Phelps ME, Kuhl DE (1979) Quantitation in positron emission computed tomography: 2 . Effects of inaccurate attenuation correction. J Comput Assist Tomogr 3:804-814.

Hurtig RR, Hichwa RD, O'Leary DS, Boles Ponto LL, Narayana S, Watkins GL, Andreasen NC (1994) Effects of timing and duration of cognitive activation in [15O]water PET studies. J Cereb Blood Flow Metab $14: 423-430$

Jensen J, McIntosh AR, Crawley AP, Mikulis DJ, Remington G, Kapur S (2003) Direct activation of the ventral striatum in anticipation of aversive stimuli. Neuron 40:1251-1257.

Kalin NH, Shelton SE, Davidson RJ, Kelley AE (2001) The primate amygdala mediates acute fear but not the behavioral and physiological components of anxious temperament. J Neurosci 21:2067-2074.
Kelly RM, Strick PL (2003) Cerebellar loops with motor cortex and prefrontal cortex of a nonhuman primate. J Neurosci 23:8432-8444.

Kim H, Somerville LH, Johnstone T, Polis S, Alexander AL, Shin LM, Whalen PJ (2004) Contextual modulation of amygdala responsivity to surprised faces. J Cogn Neurosci 16:1730-1745.

Kim JJ, Fanselow MS (1992) Modality-specific retrograde amnesia of fear. Science 256:675-677.

Koyama T, McHaffie JG, Laurienti PJ, Coghill RC (2005) The subjective experience of pain: where expectations become reality. Proc Natl Acad Sci USA 102:12950-12955.

Lang PJ, Davis M, Ohman A (2000) Fear and anxiety: animal models and human cognitive psychophysiology. J Affect Disord 61:137-159.

LeDoux JE (2000) Emotion circuits in the brain. Annu Rev Neurosci 23:155-184.

Lissek S, Dvir S, Mc Dowell DJ, Pine DS, Shaywitz E, Grillon C (2005) Pathological anxiety is associated with sustained anxiety to an unpredictably stressful context but not with phasic fear reactions to an explicit threat-cue. Paper presented at 60th Annual Meeting of the Society of Biological Psychiatry, Atlanta, GA, May.

Maren S, Fanselow MS (1995) Synaptic plasticity in the basolateral amygdala induced by hippocampal formation stimulation in vivo. J Neurosci 15:7548-7564.

Maren S, Aharonov G, Fanselow MS (1997) Neurotoxic lesions of the dorsal hippocampus and Pavlovian fear conditioning in rats. Behav Brain Res 88:261-274.

Milad MR, Quirk GJ (2002) Neurons in medial prefrontal cortex signal memory for fear extinction. Nature 420:70-74.

Nichols T, Brett M, Andersson J, Wager T, Poline JB (2005) Valid conjunction inference with the minimum statistic. NeuroImage 25:653-660.

Ongur D, Price JL (2000) The organization of networks within the orbital and medial prefrontal cortex of rats, monkeys and humans. Cereb Cortex 10:206-219.

Phelps EA, LeDoux JE (2005) Contributions of the amygdala to emotion processing: from animal models to human behavior. Neuron 48:175-187.

Phelps EA, O’Connor KJ, Gatenby JC, Gore JC, Grillon C, Davis M (2001) Activation of the left amygdala to a cognitive representation of fear. Nat Neurosci 4:437-441.

Phillips ML, Medford N, Young AW, Williams L, Williams SC, Bullmore ET, Gray JA, Brammer MJ (2001) Time courses of left and right amygdalar responses to fearful facial expressions. Hum Brain Mapp 12:193-202.

Phillips RG, LeDoux JE (1992) Differential contribution of amygdala and hippocampus to cued and contextual fear conditioning. Behav Neurosci 106:274-285.

Phillips RG, LeDoux JE (1995) Lesions of the fornix but not the entorhinal or perirhinal cortex interfere with contextual fear conditioning. J Neurosci 15:5308-5315.

Ploghaus A, Tracey I, Gati JS, Clare S, Menon RS, Matthews PM, Rawlins JN (1999) Dissociating pain from its anticipation in the human brain. Science 284:1979-1981.

Pole N, Neylan TC, Best SR, Orr SP, Marmar CR (2003) Fear-potentiated startle and posttraumatic stress symptoms in urban police officers. J Trauma Stress 16:471-479.

Sabatinelli D, Bradley MM, Fitzsimmons JR, Lang PJ (2005) Parallel amygdala and inferotemporal activation reflect emotional intensity and fear relevance. NeuroImage 24:1265-1270.

Sullivan RM, Gratton A (1999) Lateralized effects of medial prefrontal cortex lesions on neuroendocrine and autonomic stress responses in rats J Neurosci 19:2834-2840.

Talairach J, Tournoux P (1988) Co-planar stereotaxic atlas of the human brain. Stuttgart, Germany: Georg Thieme Verlag.

Walker DL, Toufexis DJ, Davis M (2003) Role of the bed nucleus of the stria terminalis versus the amygdala in fear, stress, and anxiety. Eur J Pharmacol 463:199-216.

Xu Y, Chun MM (2006) Dissociable neural mechanisms supporting visual short-term memory for objects. Nature 440:91-95.

Zink CF, Pagnoni G, Chappelow J, Martin-Skurski M, Berns GS (2006) Human striatal activation reflects degree of stimulus saliency. NeuroImage 29:977-983. 